\title{
Lymphomatoide Granulomatose - eine kurze Darstellung der Erkrankung anhand eines außergewöhnlichen Falles
}

\section{Lymphomatoid Granulomatosis - A Short Description of an Unusual Case of the Disease}

Autoren

Institute
J. Bräunlich ${ }^{1}$, H.-J. Seyfarth ${ }^{1}$, C. Gessner ${ }^{1}$, T. Gradistanac ${ }^{2}$, H. Wirtz ${ }^{1}$

Universität Leipzig, Abteilung Pneumologie

Universität Leipzig, Institut für Pathologie eingereicht 4.6.2009

akzeptiert nach Revision 14. 7. 2009

\section{Bibliografie}

Dol http://dx.doi.org/

10.1055/s-0029-1214999

Online-Publikation: 3. 11. 2009

Pneumologie 2009; 63:

697-701 @ Georg Thieme

Verlag KG Stuttgart · New York

ISSN 0934-8387

\section{Korrespondenzadresse}

Prof. Dr. Hubert Wirtz

Uniklinik Leipzig

Abteilung für Pneumologie

Liebigstr. 20

04103 Leipzig

hubert.wirtz@medizin.uni-

leipzig.de

\section{Zusammenfassung \\ $\nabla$}

Die lymphomatoide Granulomatose ist eine seltene Erkrankung mit noch unverstandener Pathogenese. Sie zählt zu den B-Zell-proliferativen Erkrankungen mit unterschiedlichem malignem Potenzial. Die Erkrankung wird als eine angiozentrische und -destruktive lymphoproliferative Erkrankung betrachtet. Pathohistologisch findet man eine EBV-Assoziation (LMP1) und die Oberflächenmarker CD20 und CD30. Die häufigsten Lokalisationen sind die Lunge, das Nervensystem, die Niere und die Leber. Wir berichten über einen 79-jährigen Mann, welcher in der Bronchoskopie eine zusätzliche Höhle oberhalb des rechten Hauptbronchus aufwies. Das Thoraxröntgen und die CT zeigten einen knotigen luftgefüllten Hohlraum im rechten Oberlappen und eine kleine Raumforderung auf der Gegenseite. Die Diagnosesicherung erfolgte chirurgisch. Wir begannen eine Chemotherapie mit Rituximab, Cyclophosphamid, Vincristin und Prednisolon (R-CVP). Drei Monate nach der Diagnosestellung verstarb der Patient an einer abszedierenden Pneumonie.

\section{Einleitung}

Die lymphomatoide Granulomatose (LYG) ist eine angiozentrische/-destruktive lymphoproliferative Erkrankung, die mit dem Ebstein-Barr-Virus assoziiert ist. Bevorzugt sind Männer zwischen 30 und 50 Jahren betroffen. Ein Befall der Lunge, der Haut, des zentralen und peripheren Nervensystemes, der Niere, der Leber und der Milz wurde beschrieben [1-5]. Die pathohistologische Trias besteht aus 1) knotigen lymphoiden Infiltraten, 2) einer Angiitis mit transmuraler Infiltration durch Lymphozyten und 3) granulomatösen Veränderungen mit zentraler Nekrose. Immunologisch werden auf den Lymphozyten CD 3, 20, 30 und

\section{Abstract \\ $\nabla$}

Lymphomatoid granulomatosis (LYG) is a rare disease with an unknown pathogenesis. It is considered as a B-cell disorder with an uncertain malignant potential. The disease is classified as an angiocentric and angiodestructive lymphoproliferative disorder. EBV-association (LMP1) and the detection of the surface antigens CD20 and CD30 are the characteristic pathological findings. The lung, the nervous system, the kidneys and the liver are affected most frequently. In the present report the case of a 79-year-old man is described, who showed a cavity in the upper right lobe which was opened out into the trachea. The initial CXR and CT scan demonstrated a large nodular air space opacity (reversed halo sign) on the upper right area and a smaller one on the left side. In addition to CT and bronchoscopy, a surgical biopsy was performed to confirm the diagnosis of lymphomatoid granulomatosis. Treatment consisted in chemotherapy with rituximab, cyclophosphamid, vincristin and prednisolone (R-CVP). Three months after confirmation of diagnosis the patient died of an abscess-forming pneumonia.

das latency membrane protein (LMP1) gefunden. Aus diesem Grunde wird die LYG den EBV-assozierten B-Zell-Lymphoproliferationen zugeschrieben ( Tab. 1).

Tab. 1 EBV-assoziierte B-Zell-Lymphoproliferation.

Burkitt's Lymphom

Klassisches Hodgkin-Lymphom

Post-Transplantations Lymphoproliferation

HIV-assoziierte Lymphoproliferation

Lymphomatoide Granulomatose

u.a.m. 


\section{Fallbeschreibung}

$\nabla$

Ein 79-jähriger Mann wurde mit den Symptomen Nachtschweiß, Fieber, Gewichtsverlust, Husten und verschlechtertem Allgemeinzustand eingewiesen. Eigenanamnestisch waren eine Sarkoidose mit kutaner Manifestation, ein Prostatakarzinom (Stadium II) und eine arterielle Hypertonie (WHO Stadium I) bekannt. In der Computertomografie des Thorax zeigten sich ein konsolidierendes alveoläres Infiltrat im rechten Oberlappen und ein kleineres im linken Oberlappen ( $\bullet$ Abb. 1). Laborchemisch fand sich eine geringe Erhöhung des C-reaktiven Proteins
(72 mg/l) ohne Leukozytose. Die Proteinelektrophorese ergab einen Doppelgradienten im Bereich der Gammaglobuline (Gamma 15,9\%; Grad 12,1 ; Grad 2 3,4) und eine Doppelbande in alpha2 $(13,4 \%)$. Es erfolgte eine Bronchoskopie mit transbronchialer Biopsie, deren histologische Aufarbeitung mit Nachweis von lymphozytären Infiltraten und herdförmigen Nekrosen zur Verdachtsdiagnose einer lymphomatoiden Granulomatose Grad II bis III führte ( Abb.5-7). Auffällig war ein zusätzliches Ostium oberhalb des Abganges des rechten Hauptbronchus, welches in einen Hohlraum führte ( $\bullet$ Abb. 1-4).
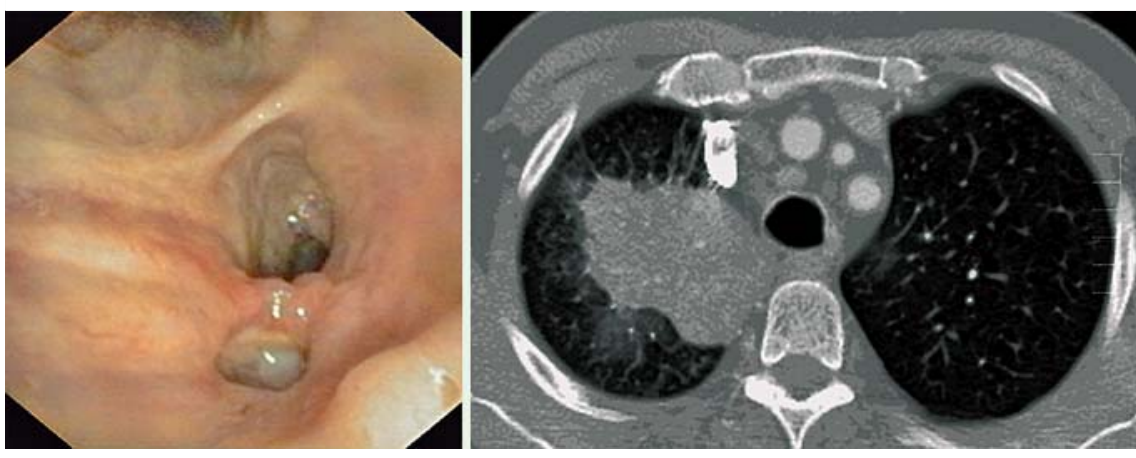

Abb. 1 Aufnahmetag / CT 2 Wochen zuvor angefertigt.

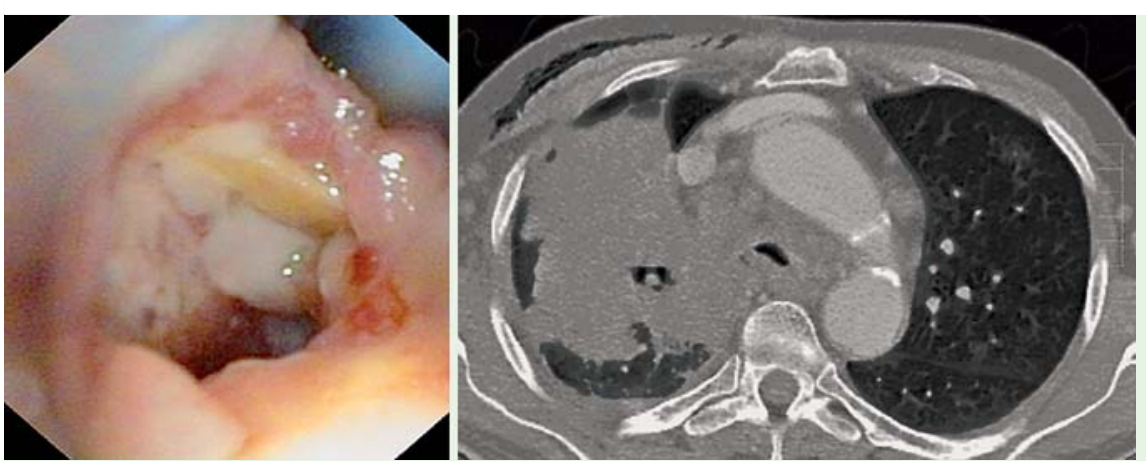

Abb. 2 Restaging 4 Wo. nach Erstkonsultation.
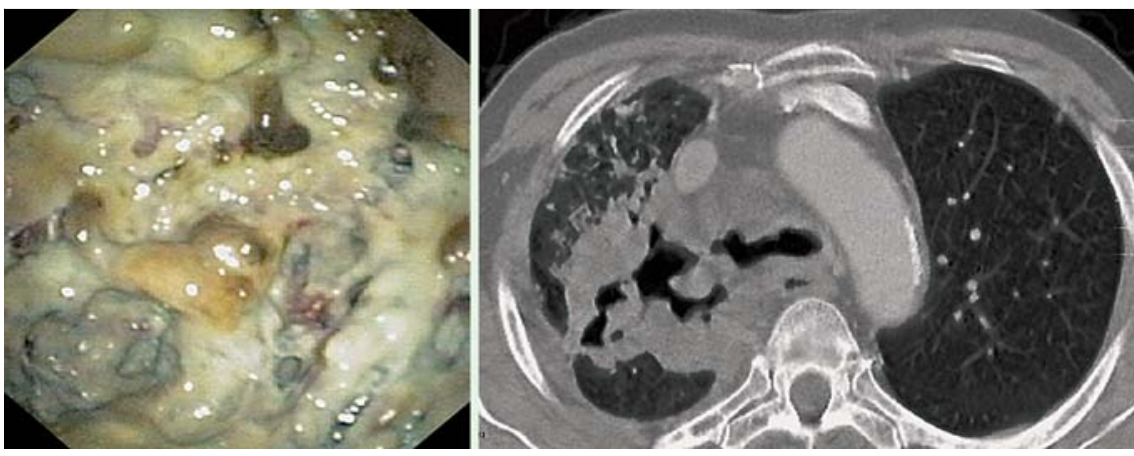

Abb. 3 Restaging 8 Wo. nach Erstkonsultation.
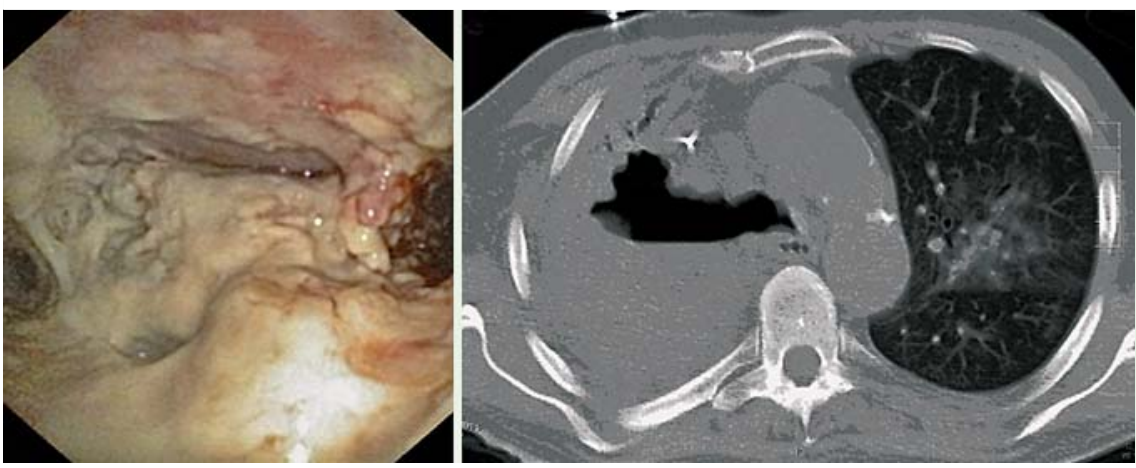

Abb. 4 Bronchoskopie / CT 2 Tage prämortal. 


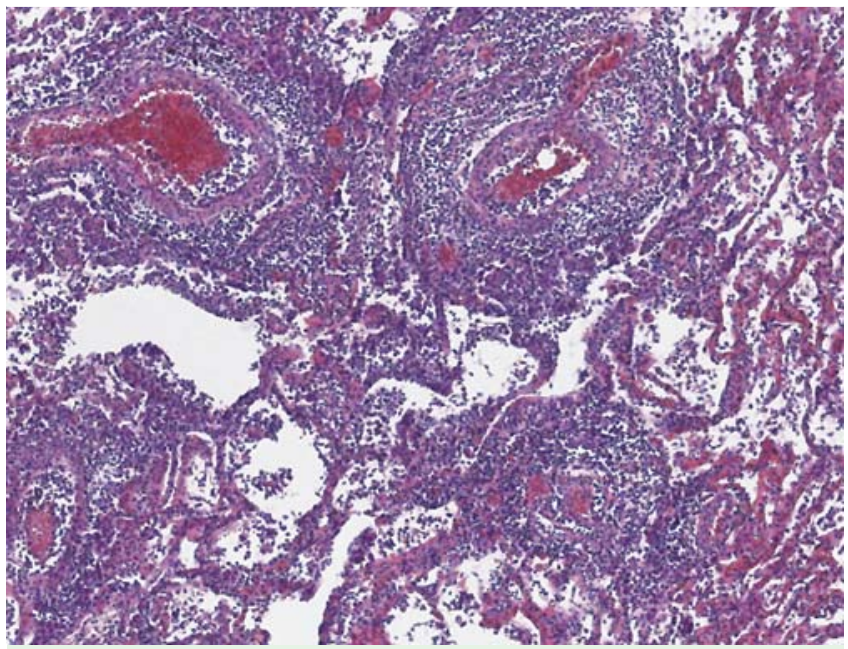

Abb. 5 Lunge $10 \times$ T-Lymphozyten mit Gefäßwandinfiltration.

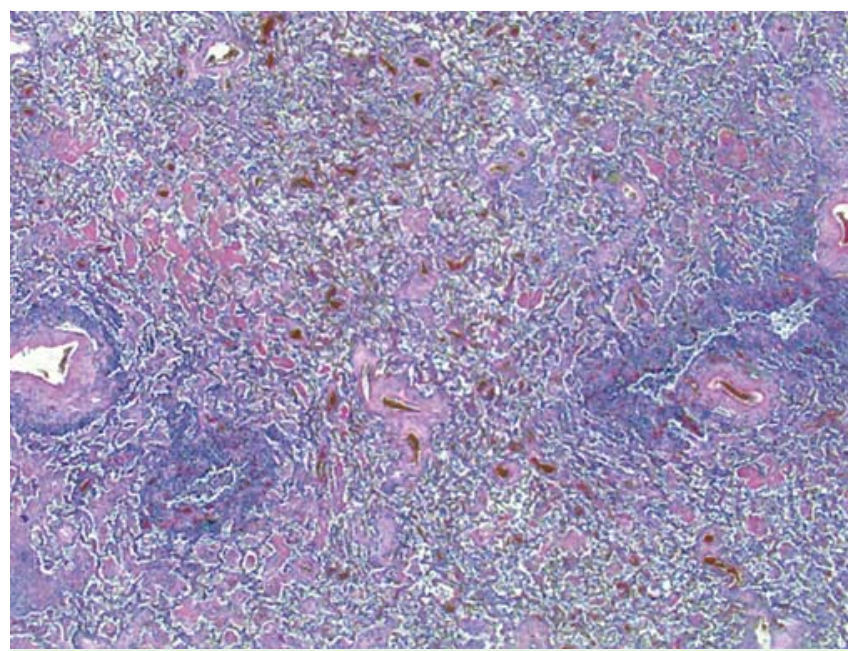

Abb. 6 Lunge 2,5 × T-Lymphozyten mit Gefäßwandinfiltration, fibringefüllte Alveole, Bronchusinfiltration.

Die bronchoalveoläre Lavageflüssigkeit (BALF) ergab ein Zellbild mit erhöhtem Lymphozyten- (15\%) und neutrophilen Granulozytenanteil (10\%), einen erniedrigten CD4/CD8-Quotienten $(0,52$; CD4 27\%/ CD8 52\%) und einen erhöhten Anteil der zytotoxischen CD57+CD8+-T-Zellen (26\%). Insgesamt zeigte die BALF eine vermehrte Aktivität der T-Zellen. Zusätzlich waren EBV-Genomäquivalente im Serum (1048 Genomäquvalente/ml) und in der BALF

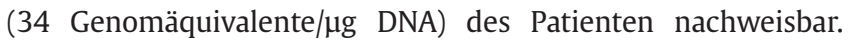
Nachfolgend wurde eine Video-assistierte Thorakoskopie (VATS) durchgeführt. Hier konnte der Verdacht auf eine lymphomatoide Granulomatose bestätigt werden ( $\bullet$ Abb. 5-7). Das Staging, welches die Biopsie einer auffälligen Hautläsion einschloss, ergab keine weiteren Manifestationen der Erkrankung. Es wurde eine Kombinationstherapie mit Rituximab ( $375 \mathrm{mg} / \mathrm{m}^{2}$ KÖF, Tag 1), Cyclofosfamid (400 mg, Tag 2), Vincristin (1 mg, Tag 2) und Prednisolon (100 mg Tag 1 und Tag 2) begonnen. Infolgedessen war eine kurzfristige Besserung der subjektiven Symptomatik, insbesondere ein Nachlassen des Nachtschweißes, zu beobachten. Kurz vor dem 2. Zyklus Chemotherapie erkrankte der Patient an einer Pneumonie unter Immunsuppression ( $\boldsymbol{A} \mathbf{A b b}$. 8). In der bronchoalveolären Lavageflüssigkeit (BALF) ließ sich Pseudomonas aeruginosa $(100000 \mathrm{KBE} / \mathrm{ml})$ nachweisen. Trotz Eskalation der Therapie mit intravenöser Gabe von Imipenem, Moxifloxacin

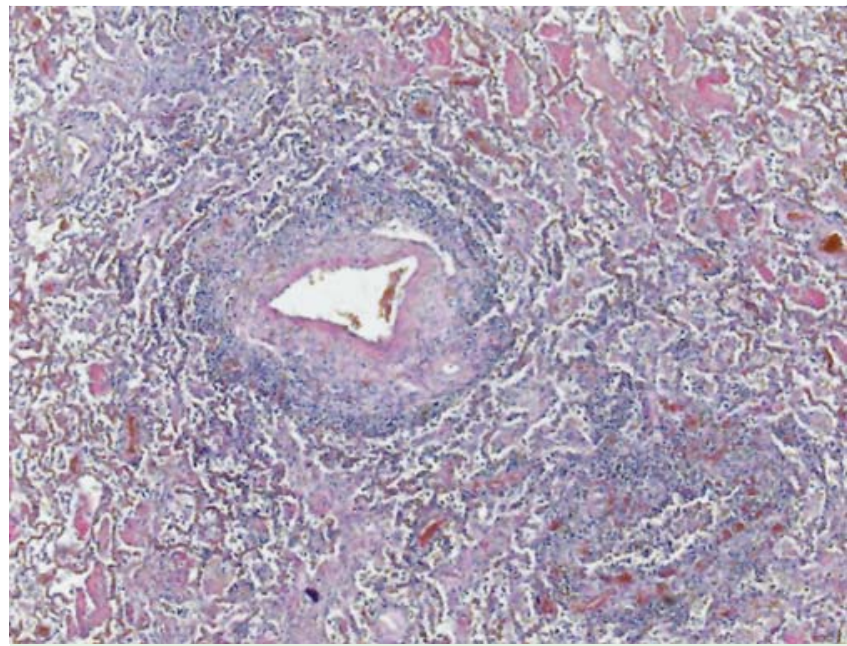

Abb. 7 Lunge $5 \times$ Gefäßwandinfiltration.

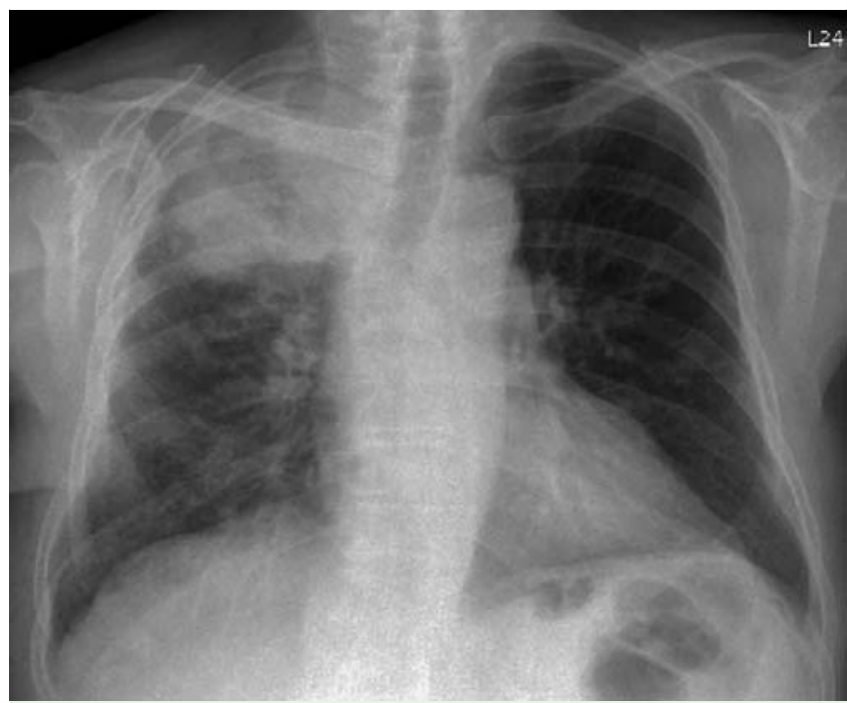

Abb. 8 Pneumonie vor dem 2. Zyklus Chemotherapie.

und Inhalation von Tobramycin konnte die Infektion nicht beherrscht werden. Bildmorphologisch und bronchoskopisch war darüber hinaus ein deutlicher Progress der lymphomatoiden Granulomatose zu beobachten ( $\bullet$ Abb. 2, 3). Der Patient verstarb unter dem Bild einer abszedierenden Pneumonie ( $\bullet$ Abb. 4). Die Obduktion bestätigte die radiologischen und klinischen Befunde und zeigte einen ausgeprägten Progress der Grunderkrankung.

\section{Diskussion \\ $\nabla$}

Epidemiologie und Pathogenese

Die Erstbeschreibung des Krankheitsbildes lymphomatoide Granulomatose erfolgte durch Liebow et al. 1972 [6]. Erste Untersuchungen wurden 1979 durch Katzenstein et al. [7] durchgeführt. Insgesamt wurden mehr als 600 Fälle beschrieben. Man findet in der Literatur aufgrund der Seltenheit der Erkrankung keine validen Angaben zur Inzidenz. Häufig sind Männer zwischen dem 30. und 50. Lebensjahr betroffen. Die Letalität liegt bei $50 \%$. Spontanremissionen werden in $20 \%$ der Fälle beobachtet. Die mittlere Überlebenszeit liegt bei 14-72 Monaten. Die Erkrankung kann assoziiert sein mit dem Sjögren-Syndrom, der chronischen Hepatitis, der rheumatoiden Arthritis, einer Nierentransplantation 


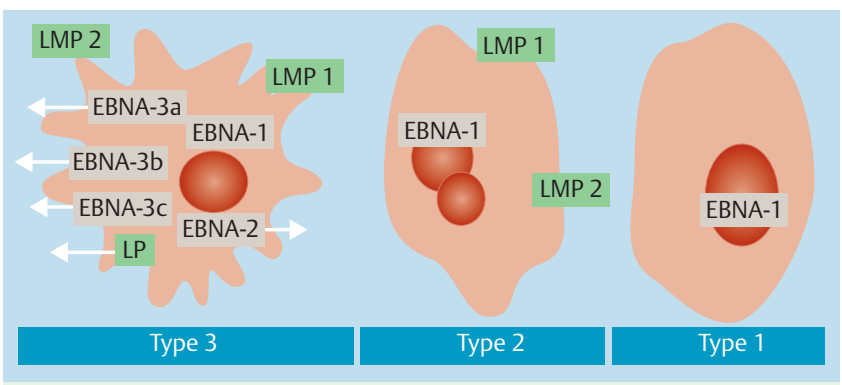

Abb. 9 Latenztypen des EBV nach Heslop 2005 [11].

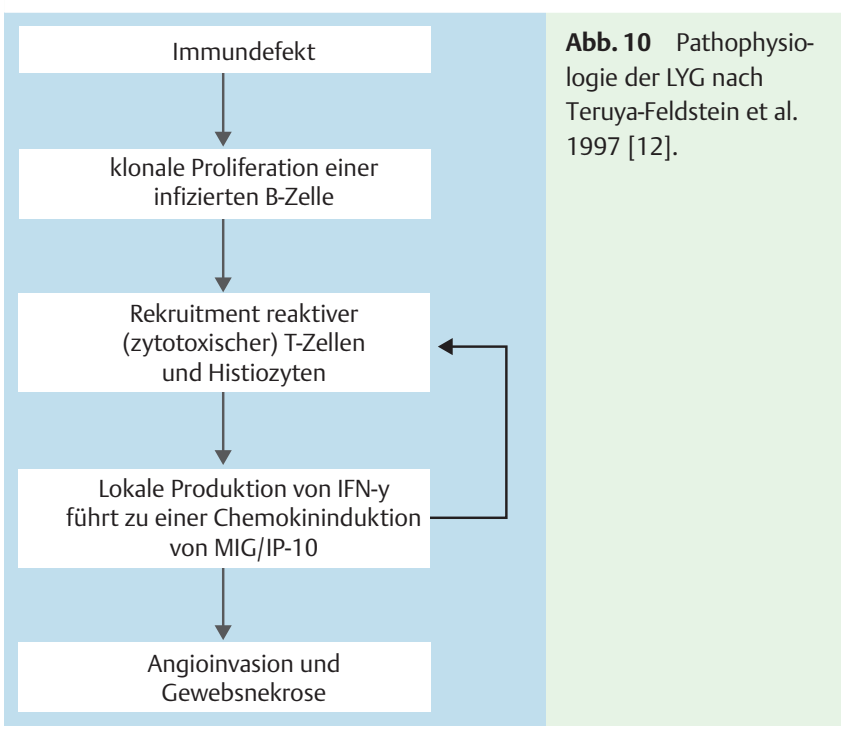

und einer HIV-Infektion. Auch Patienten mit Abnormitäten der T-Zellen zeigen ein erhöhtes Risiko [9].

Zunächst erfolgte aufgrund der T-Zell-vermittelten Effekte der Angioinvasion und -destruktion die Einordnung als T-Zell-lymphoproliferative Erkrankung [9]. Entscheidend waren die späteren Erkenntnisse über die EBV-Assoziation [10] und die klonale Proliferation der betroffenen B-Zellen. Basierend auf den bisherigen Erkenntnissen kann man die Erkrankung als ein T-Zell-reiches B-Zell-Lymphom betrachten [1]. Pathophysiologisch ursächlich ist die Latenzform 2 des Ebstein-Barr Viruses [11]. Kennzeichnend hierfür ist die Expression der Latenzproteine (latency membrane protein $1+2$, LMP), des nukleären Antigenes (EBNA) und der small non-coding EB-RNA (EBER). Den Typ 1 findet man am häufigsten beim Burkitt-Lymphom, den Typ 3 bei der HIV-Infektion ( Abb. 9) [11].

Die Bindung des EBV an den CD21-Rezeptor ist die Voraussetzung für die infizierte B-Zelle, im Verlaufe maligne Potenz zu erlangen. In vivo wird diese durch die Immunreaktion kontrolliert. Infolge eines Immundefektes kommt es zu einer klonalen Proliferation der infizierten B-Zelle. Unter der Vermittlung von IL-12 und TNF- $\alpha$ werden reaktive T-Zellen aktiviert. Die nun einsetzende lokale Produktion von Interferon- $\gamma$ aktiviert die Chemokine MIG

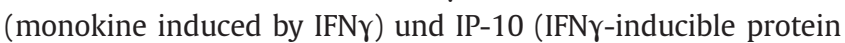
10) ( Abb. 10) [12], welche vom umgebenen Gewebe ausgeschüttet werden.
Tab. 2 Symptomatik und Diagnostik.

Lungen
Luftnot, Husten, produktives Sputum, Hämoptysis
B-Zell-Symptomatik (Fieber, Nachtschweiß, Gewichtsverlust)
Haut
schmerzhafte erythematöse, selten ulzeriertende Papeln/Macula
gluteal und an den Extremitäten
PNS/ZNS
- neurologische Symptomatik (Bewusstseinstörungen, Ataxie,
Hemiparese und Krämpfe)
- sensorische Neuropathie/Mononeuritis multiplex
Niere
Leber

Tab. 3 Einteilung der LYG.

Grad I
- polymorphes angiozentrisches Infiltrat ohne auffällige lymphozytäre
Atypie
- minimale bis nicht nachweisbare Nekrosen
- Zahl der EBV-Zellen weniger als 5/HPF oder nicht vorhanden
Grad II
- Angioinvasion und/oder -destruktion, verstreut liegend
- große oder hyperchromatische lymphoide Zellen
- weniger als 20 EBV-Zellen/HPF
- Nekrosezeichen wenig ausgeprägt
Grad III (großzelliges B-Zell-Lymphom)
- große atypische lymphoide Zellen in polymorphem Umfeld
- EBV-Zellen mehr als 20/HPF
- ausgedehnte konfluente Nekrosen

\section{Symptomatik und Diagnostik}

Aus dem Befall unterschiedlicher Organe resultiert die Klinik $(\bullet$ Tab.2). Häufig werden die Patienten durch eine pulmonale Symptomatik (50\% der Fälle), eine B-Symptomatik und im Rahmen radiologischer Zufallsbefunde auffällig. Im Falle des Patienten waren dies der Husten und die B-Symptomatik. Eine chirurgische Sicherung ist obligat und wurde auch bei unserem Patienten durchgeführt, da der pathohistologische Befund der transbronchialen Biopsie nur eine Verdachtsdiagnose formulieren konnte. Oftmals können im Serum, in der BALF, wie im Falle des hier beschriebenen Patienten, und im Resektat EBV-Genomäquivalente gefunden werden. Pathohistologisch ist die LYG durch knotige lymphoide Infiltrate, eine transmurale Angiitis, zentral nekrotische Granulome und den Nachweis von CD 3, 20, 30 und LMP (latency membrane protein $1+2$ ) charakterisiert. Die Einteilung erfolgt in drei Schweregrade. Der Grad 3 entspricht einem großzelligen B-Zell-Lymphom ( $\bullet$ Tab. 3).

Laborchemisch existieren keine typischen Veränderungen. Es können eine Leukopenie oder eine Leukozytose auftreten. Organspezifische Parameter zeigen sich manchmal leicht verändert, jedoch ohne wegweisenden Charakter. Die durchflusszytometrische Untersuchung der BALF erbringt häufig einen erniedrigten CD4/CD8-Quotienten. Die radiologische Diagnostik zeigt abnormale, jedoch unspezifische Befunde. Differenzialdiagnostisch müssen Pseudolymphome, maligne Lymphome, lymphozytäre interstitielle Pneumonien, Metastasen, die Wegner Granulomatose, die COP u.a.m. in Betracht gezogen werden. Häufige bildmorphologisch dokumentierbare Veränderungen sind: 
- bilaterale Raumforderungen in den unteren oder peripheren Lungenabschnitten (80-100\%)

- Pleuraergüsse $(33 \%)$

- Alveolitis (30\%)

- Kavernen (30\%)

- Pneumothorax $(5 \%)$

\section{Therapie}

$\nabla$

In der Literatur existieren Dokumentationen unterschiedlicher Therapien und Behandlungserfolge. Es wurden Heilungen und stabile Krankheitsverläufe unter Prednisolon, Interferon-alpha2, Ganciclovir und verschiedenen Zytostatikakombinationen berichtet [2,14-19]. Zum jetzigen Zeitpunkt sind folgende Therapieoptionen möglich:

1. gutartiger Verlauf: keine Therapie (spontane Remissionen)

2. symptomatisch/progressiv:

Kortikosteroide mit/ohne Chemotherapeutika

3. high-grade Lymphom mit Chemotherapie

4. Radiotherapie/chirurgische Resektion bei lokalisiertem Befall

5. Ganciclovir, Interferon-alpha-2

Die Auswahl der Therapie muss nach der Schwere des Befalls, des Grades der LYG und des Zustandes des Patienten abgewogen werden. Leider existieren aufgrund der Seltenheit der Erkrankung bis heute keine einheitlichen Therapiekonzepte. Die bislang größte Studie mit 152 Patienten konnte keine signifikanten Unterschiede zwischen der Mortalität und dem krankheitsfreien Überleben bei verschiedenen Therapieoptionen detektieren [7]. Jedoch kann bei Vorliegen eines Grades I oder eines gering ausgeprägten lokalisierten Befundes aufgrund der hohen Spontanremissionsrate eine abwartende Strategie verfolgt werden. Bei Progress und lokalisiertem Befall ist die Möglichkeit einer chirurgischen Resektion oder einer Radiotherapie als Therapiekonzept zu prüfen. Im Falle eines Grades II bis III oder dem Vorliegen einer multiplen Ausbreitung ist eine Chemotherapie unumgänglich.

Im Falle des hier geschilderten Patienten wurde eine Chemotherapie aufgrund des Vorliegens eines Grades II bis III (großzelliges B-Zell Lymphom) gewählt. Im Hinblick auf das fortgeschrittene Alter wurde die Dosierung der Chemotherapie reduziert. Aus demselben Grunde und wegen der uneinheitlichen Datenlage verzichteten wir auf den Einsatz einer antiviralen Therapie. Ebenso wurde die Indikation zur operativen Sanierung geprüft. Jedoch schied diese Möglichkeit wegen des beidseitigen Lungenbefalls und der ungünstigen trachealen Lage des zusätzlichen Ostiums direkt oberhalb des Abganges des rechten Hauptbronchus aus.

\section{Zusammenfassung}

$\nabla$

Der hier dargestellte Fall demonstriert die Symptomatik, Diagnostik und die Prognose einer gesicherten lymphomatoiden Granulomatose Grad II bis III. Diese seltene Erkrankung zeigt sich oftmals durch eine B-Symptomatik und radiologische Veränderungen und erfordert eine chirurgische Sicherung. Sekundäre Symptome sind Hämoptysen und opportunistische Infektionen. Es existiert derzeit noch kein einheitliches und stadienbasierendes Therapiekonzept, jedoch konnten bereits einige Heilungen bei Vorliegen eines lokalisierten und niedriggradigen Befalls erzielt werden. Der hier vorgestellte Fall dokumentiert das Scheitern einer auf den Erfahrungen der Lymphombehandlung basierenden Therapie. Komplizierend kamen durch das Vorhandensein eines Hohlraumes mit Anschluss an das Bronchialsystem ständig wiederkehrende Infektionen hinzu. Ebenso muss ein Fortschreiten der Grunderkrankung unter Therapie dokumentiert werden. Weitere Studien und Untersuchungen über Pathophysiologie und Therapien sind zur Verbesserung der Behandlung dieser Erkrankung erforderlich.

\section{Interessenkonflikte}

Die Autoren erklären, dass keine Interessenkonflikte im Sinne der Richtlinien des International Committee of Medical Journal Editors bestehen.

\section{Literatur}

1 McNiff JM et al. Lymphomatoid granulomatosis of the skin and lung. An angiocentric T-cell-rich B-cell lymphoproliferative disorder. Arch Dermatol 1996; 132: $1464-14670$

2 Jordan $\mathrm{K}$ et al. Successful treatment of mediastinal lymphomatoid granulomatosis with rituximab monotherapy. Eur J Haematol 2005; 75: $172-173$

3 Okuda T et al. A case of lymphomatoid granulomatosis followed for 14 months on the basis of clinical and histological findings. Brain Tumor Pathol 2008; 25: 33 - 38. Epub 2008 Apr 16

4 Cachat $\mathrm{F}$ et al. Lymphomatoid granulomatosis in a renal transplant patient. Pediatr Nephrol 2003; 18: 838 - 842. Epub 2003 Jun 12

5 Schjølseth SA, Blom GP. Lymphomatoid granulomatosis of the lung, liver and spleen. Scand J Haematol 1978; 21: 104-108

6 Liebow AA et al. Lymphomatoid granulomatosis. Hum Pathol 1972; 3: 457-558

7 Katzenstein AL. Lymphomatoid granulomatosis: a clinicopathologic study of 152 cases. Cancer 1979; 43: 360 - 373

8 Kamangar N. 2006; www.emedicine.com

9 Nonomura A. T cell lymphoma presenting clinical and morphological features resembling polymorphic reticulosis and lymphomatoid granulomatosis. Acta Pathol Jpn 1983; 33: 1289-1301

10 Katzenstein AL, Peiper SC. Detection of Epstein-Barr virus genomes in lymphomatoid granulomatosis: analysis of 29 cases by the polymerase chain reaction technique. Mod Pathol 1990; 3: 435-441

11 Heslop HE. Biology and treatment of epstein-barr virus-associated non-hodgkin lymphomas. Hematology Am Soc Hematol Educ Program 2005; $1: 260-266$

12 Teruya-Feldstein J et al. The role of Mig, the monokine induced by interferon-gamma, and IP-10, the interferon-gamma-inducible protein10, in tissue necrosis and vascular damage associated with EpsteinBarr virus-positive lymphoproliferative disease. Blood 1997; 90: 4099-4105

13 Cadranel J et al. Primary pulmonary lymphoma. Eur Respir J 2002; 20 : $750-762$

14 Richter C et al. Lymphomatoide Granulomatose - Remissionsinduktion mit Interferon alpha-2b. Dtsch Med Wochenschr 1997; 122: 11061110

15 Oosting-Lenstra SF et al. Failure of CHOP with rituximab for LYG. Neth J Med 2007; 65: $442-447$

16 Fisseler-Eckhoff $A$. et al. Differenzialdiagnose multipler Rundherde, lymphomatoide Granulomatose. Pathologe 1996; 17: 301 - 304

$17 \mathrm{Yu}-\mathrm{Hui} \mathrm{H}$ et al. Successful treatment of elderly advanced lymphomatoid granulomatosis with rituximab-CVP combination therapy. Eur J Haematol 2007; 78: 176-177

18 Jaffre S et al. Fatal haemoptysis in a case of lymphomatoid granulomatosis treated with rituximab. Eur Respir J 2006; 27: 644-646

19 Polizzotto $M N$ et al. Failure of rituximab monotherapy in lymphomatoid granulomatosis. Eur J Haematol 2005; 75: 172 - 173

20 Lipford EH et al. Angiocentric immunoproliferative lesions: a clinicopathological spectrum post-thymic T-cell proliferations. Blood 1988; 72: $1674-1681$ 